\title{
TRANSPORT AND MAGNETIC PROPERTIES OF RARE EARTH COMPOUNDS EPITAXIALLY GROWN ON SEMICONDUCTORS
}

\author{
JaN Chroboczek
}

Centre National d'Etudes des Télécommunications, 38243 Meylan, France

\begin{abstract}
Growth, crystallographic structure, electrical transport and magnetic properties of cubic Er pnictides and hexagonal Er silicide layers, epitaxially grown on semiconductors, are reviewed. Magnetoresistance anomalies observed at low temperatures are discussed in terms of a model developed for electron-magnetic moment interactions in antiferromagnetic systems.
\end{abstract}

PACS numbers: 72.20.My, 68.55.-a, 75.50.Pp

\section{Introduction}

The Rare Earth (RE) elements remained outside the realm of semiconductor physics until very recently. About a decade ago first attempts were made $[1,2]$ to imbed the RE atoms in semiconductors. Because the optically active transitions in RE occur within a deep, hence a well-screened ( $4 f$ ) atomic layer, they are not strongly affected by the crystalline surroundings and it has been hoped that many of the attractive optical properties [3] of RE could be exploited in semiconductors, with the help of all the riches of the semiconductor technology and the know-how. One of the most attractive was the perspective of creating direct transitions in non-direct gap semiconductors, silicon being, of course, the main target. The interest in Er can be linked to the fact that the energy spacing between the first excited state and the ground state of $\mathrm{Er}^{+3}$ matches that of the optical window of silica fibres $(0.8 \mathrm{eV})$. Despite the considerable efforts, the RE-doped semiconductors have not proven to be interesting enough for optical applications and the related optoelectronic studies seem to be diminishing now in intensity. At the same time studies of RE compound layers on semiconductors become increasingly popular. There are several reasons for that. First, all the known RE compounds that can be epitaxially grown on semiconductors are metallic and form smooth interfaces, whose tunneling properties present considerable interest. Some of the 
heterojunctions have unusually low barrier heights, which, in conjunction with the metallic properties of the RE compounds make the latter attractive for interconnections in microelectronics. Junctions with more elevated barriers can be used for controlled tunneling and can find applications in optoelectronics. The second area of interest is that of magnetic properties of the RE compounds, associated with a large value of the magnetic moment of the $4 f$ layer of $R E$ elements. These properties are unusual and can be studied by electrical transport methods, because the magnetic ions strongly interact with conduction electrons. A dominant feature in magnetic properties of the RE-compound layers deposited on semiconductors, studied so far, is the antiferromagnetic component. It is therefore doubtful that the $\mathrm{RE}$ compounds layers can find application in devices, composed of high mobility semiconductors and magnetic layers [4]. However, applications in devices employing the coupling of the magnetic vector of the electromagnetic radiation with $\mathrm{RE}$ spins are envisagible.

In this paper we shall discuss two classes of RE compounds epitaxially grown on semiconductors: (i) cubic RE pnictides (RE-V) grown on III-V compounds, and (ii) hexagonal RE silicides grown on (111) surface of $\mathrm{Si}$.

\section{Growth and characterization 2.1. RE pnictides}

The RE-V compounds have the $\mathrm{NaCl}$ structure and are stoichiometric. The RE-As has been explored first, because of its thermodynamical stability, permitting the use of elevated temperatures for the vacuum deposition, facilitating both, the ordering and the elimination of the As excess [5-7]. The substrate materials are, usually, GaAs and InP.

\section{TABLE I}

Lattice constants of some $\mathrm{Er}-\mathrm{V}$ compared with GaAs and InP.

\begin{tabular}{c|c|c|c|c|c}
\hline \hline Compound & ErP & GaAs & ErAs & InP & ErSb \\
$a(\AA)$ & 5.606 & 5.653 & 5.745 & 5.869 & 6.107
\end{tabular}

Table I lists, in ascending order, the lattice constants of some RE-V and the substrates, showing that it is possible to form RE- $V$ ternary compounds with a perfect lattice matching with respect to the substrates. The matched alloys are ternary and are obtained [6] from the RE-V flanking the two respective substrate materials in Table I. Another method of obtaining lattice-matched heterojunctions consists of using two RE elements with one group V element [7].

\section{2. $R E$ silicides}

The RE silicides form tetragonal and hexagonal $\mathrm{AlB}_{2}$ structures and are not stoichiometric. They are usually prepared by contact reaction method following the vacuum deposition of $\mathrm{RE}$ on the cleaned $\mathrm{Si}$ (111) surface [8]. This surface 
orientation favours the formation of the hexagonal silicide which, thanks to its planar geometry, with $\mathrm{Si}$ and $\mathrm{Er}$ atoms separated into parallel sheets, should give continuous and uniform layers. However, the layers are rough, unless rapid thermal annealing is used, the method that has been demonstrated [9] to yield hexagonal monocrystalline films. The best films so far obtained, were MBE-fabricated, by codeposition of $\mathrm{Er}$ and $\mathrm{Si}$ in the ratio close to the stoichiometry of the disilicide, followed by a solid state reaction [10]. The crystallographic properties of the silicide are similar to those of bulk $\mathrm{ErSi}_{2}$. The projection of the structure on (0001) plane is shown schematically in Fig. 1 where the separation of $\mathrm{Er}$ and $\mathrm{Si}$ atoms into distinct sheets is clearly visible. High energy electron diffraction (HEED) pattern reveals

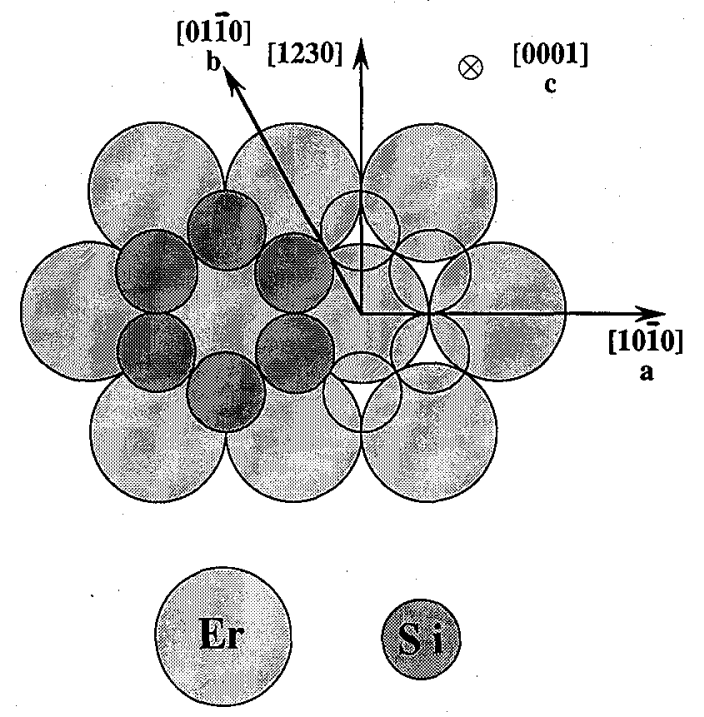

Fig. 1. Crystallographic structure of Er silicide.

(Fig. 2(a)) existence of a superstructure in the arrangement of Si atoms, interpreted as a periodic arrangement of $\mathrm{Si}$ vacancies in the lattice [9]. The HEED pattern may differ in details, depending on a preparation method and stoichiometry, and that presented in Fig. 2(a) is characteristic for the MBE fabricated [10] $\mathrm{ErSi}_{1.7}$, in whose structure one atom is missing, on the average, in every hexagonal ring of Si atoms. The TEM picture, shown in Fig. 2(b), revealed the existence of fringes corresponding to the periodicity given by HEED. It should be noted that the $\mathrm{Si}$ vacancy arrangement has been found in hexagonal $\mathrm{Tb}$ and $\mathrm{Y}$ disilicides with $\mathrm{Si}$ deficiency $[11,12]$. The vacancy formation problem has not yet been studied in detail and no systematic comparison with corresponding bulk crystals is available. The vacancy formation might be linked to biaxial stress in the films and/or to the planarization of $\mathrm{Si}$ atoms in sheets compressed between the RE (or Y) ions, entailing a reduction in interatomic spacings between $\mathrm{Si}$ atoms as compared to the spacings on the (111) surface of Si. The vacancy formation is certainly one of the subjects that merits more research efforts. 

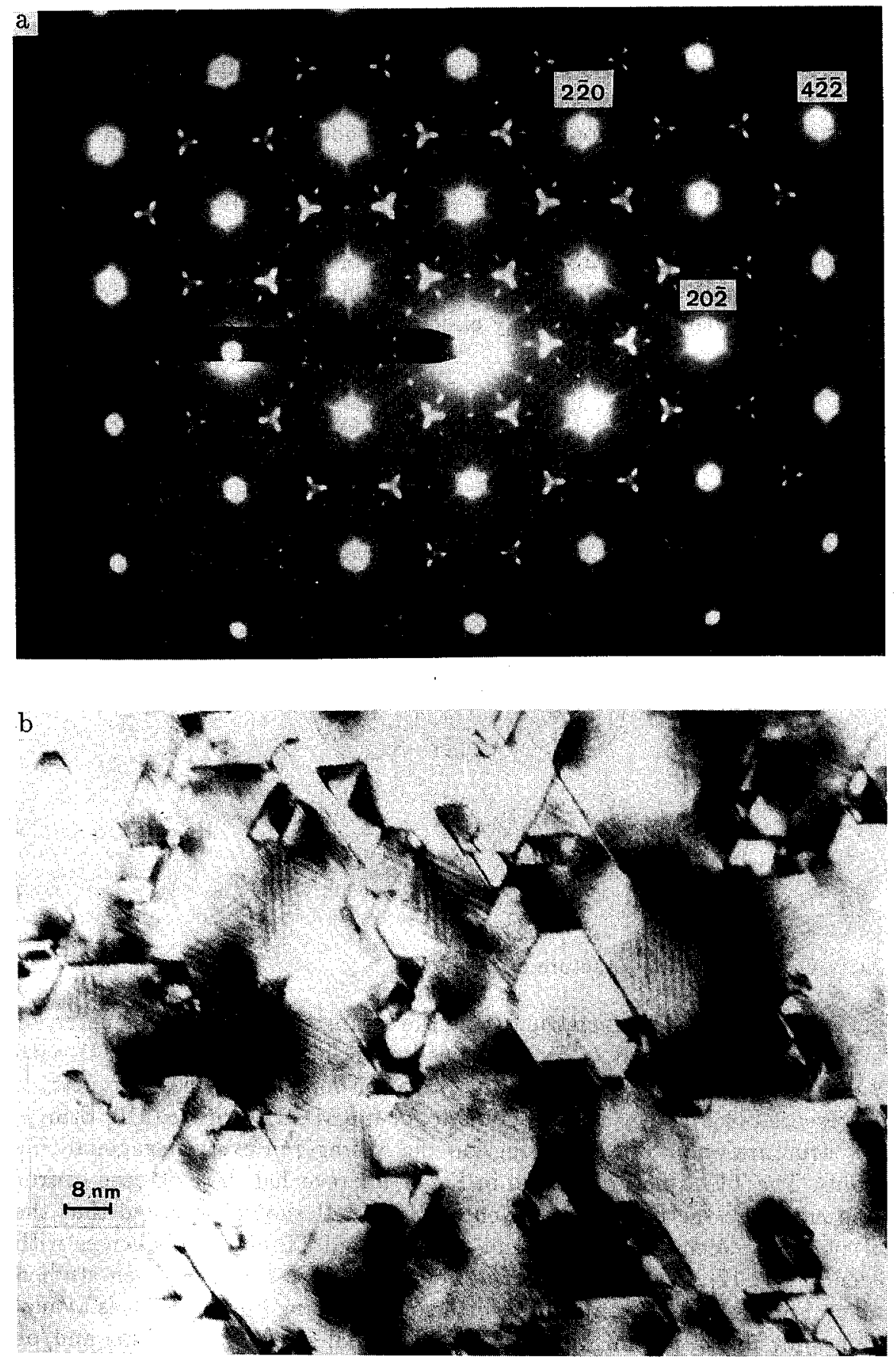

Fig. 2. (a) High energy diffraction pattern of Er silicide for the [111] orientation of Si. The weak spots originate from the superstructure of vacancies. (b) The supestructure shown in (a) gives a system of fringes in direct image of TEM. 


\section{Electrical transport properties}

All the known $\mathrm{Er}$ compounds show metallic properties and their resistivity $\rho$ follows the Matthiesen law,

$$
\rho=\rho_{0}+\text { const } \cdot T,
$$

down to the temperature $T_{\mathrm{c}}$ of magnetic ordering of the RE spins.

TABLE II

Some characteristics of Er compounds.

\begin{tabular}{l|c|c|c|c|c}
\hline \hline Compound & $\begin{array}{r}\varrho(300 \mathrm{~K}) \\
(\mu \omega \mathrm{cm})\end{array}$ & $\begin{array}{c}\varrho\left(T_{\mathrm{c}}\right) \\
(\mu \omega \mathrm{cm})\end{array}$ & $\begin{array}{c}T_{\mathrm{c}} \\
(\mathrm{K})\end{array}$ & $\begin{array}{c}B_{\mathrm{c}} \\
(\mathrm{T})\end{array}$ & Reference \\
\hline $\mathrm{Er}_{3} \mathrm{Si}_{5}$ & 35 & 9.5 & 4.3 & 1.8 & {$[10]$} \\
$\mathrm{ErP}$ & 155 & 86.5 & 4.8 & 0.5 & {$[14]$} \\
$\mathrm{ErSb}$ & 48 & 20.0 & 4.4 & 1.0 & {$[14]$} \\
$\mathrm{ErAs}$ & 39 & 9.7 & 4.5 & 9.9 & {$[13]$} \\
$\mathrm{ErAs}_{0.4} \mathrm{P}_{0.6}$ & 95 & 26.5 & 3.1 & 0.5 & {$[14]$} \\
$\mathrm{Er}_{0.7} \mathrm{Sc}_{0.3} \mathrm{As}$ & 44 & 14 & $?$ & $?$ & {$[15]$}
\end{tabular}

Table II lists some of the electrical properties of Er compounds. Note that $\mathrm{ErSi}_{1.7}$ and ErAs have the lowest resistivities and the most elevated ratio of the room temperature to the low temperature resistivities. Figures 3(a) and 3(b) show $\rho$ and the Hall coefficient $R_{\mathrm{H}}$ plotted vs. $T$. While $\rho$ decreases linearly down to $T_{\mathrm{c}}$ (where it drops, see inset), $R_{\mathrm{H}}$ first decreases linearly, changing sign at around $150 \mathrm{~K}$, to increase in value near $30 \mathrm{~K}$. The change of sign of $R_{\mathrm{H}}$ is characteristic for compensated semimetals, where the electrons and the holes coexist. Our low $T$ magnetoresistance (MR) data have not revealed yet the Shubnikov-de Haas oscillations and no calculations on the Fermi surface of Er silicide exist. The Fermi surface should consists, however, of segments possessing the hole-like curvature and also the electron pockets. Some of them should intersect the Brilloin zone boundary, because a linear magnetoresistance is observed at high magnetic fields (CNET results, unpublished), characteristic for open orbit transport. A recent paper [15] on properties of $\mathrm{Er}_{0.7} \mathrm{Sc}_{0.3}$ As buried in $\mathrm{GaAs}$ (a compound with a perfect match to the substrate), brought a first report on the Shubnikov-de Haas oscillations in Er compounds. The data show that the Fermi surface is composed of a sphere near the point and electron pockets at $\mathrm{X}$ points of the Brillouin zone. Further improvement in the crystal quality in other compounds is needed for the Fermi structures studies, and the lattice-matched compounds are probably the most promising.

Two low temperature features of $\varrho(T)$ and $R_{\mathrm{H}}(T)$ plots shown in Fig. 3, should be pointed out. First is the low temperature resistance anomaly, shown in the expanded scale the inset in Fig. 3(a), associated with the paramagnetic-antiferromagnetic phase transition and a subsequent suppression of disorder in the spin system, when $T$ is lowered below $T_{\mathrm{c}}$ (cf. following section for discussion). Figure 4 shows that the resistivity anomaly occurs in all the Er compounds at temperatures 
a

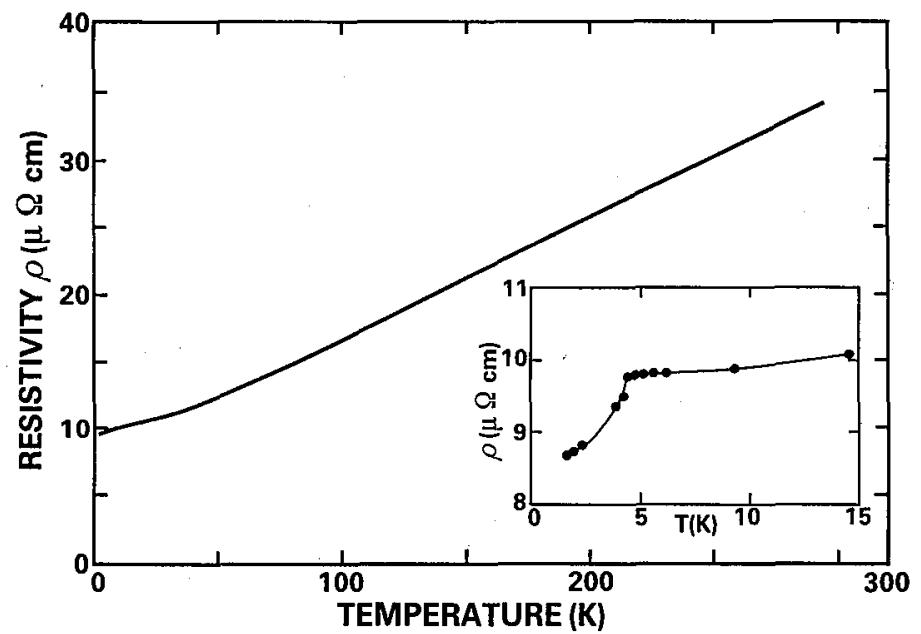

$\mathrm{b}$

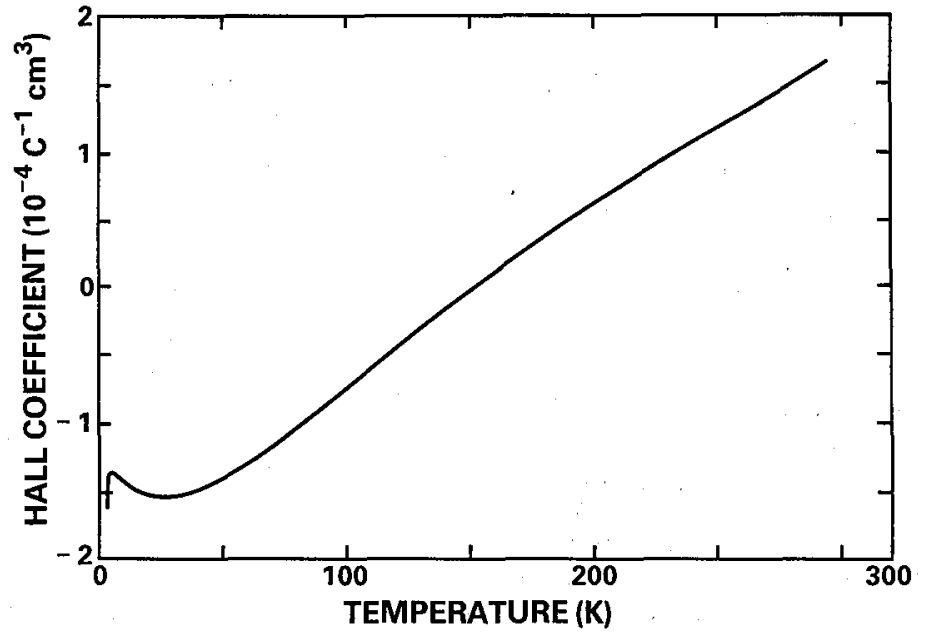

Fig. 3. (a) Resistivity of a $300 \AA$ thick Er silicide film on $\mathrm{Si}$ (111), as a function of temperature. The inset shows the low temperature part where magnetic ordering of $\mathrm{Er}$ spins induces a resistivity drop. (b) Hall coefficient of the same sample. Note the sign change at about $150 \mathrm{~K}$ and an increase of the coefficient below $30 \mathrm{~K}$.

close to $5 \mathrm{~K}$ (except for the ternary RE-V-V', where it is lower, probably because of the alloy scattering contribution). The second low-temperature feature is that of the $R_{\mathrm{H}}$ increase near $25 \mathrm{~K}$, shown in Fig. 3(b). In several more recent samples the increase in $R_{\mathrm{H}}$ was observed at $T$ as high as $100 \mathrm{~K}$, with a very strong increase of $R_{\mathrm{H}}$ in the absolute value. The effect, that has also been studied in other magnetic materials, is attributed to the increase of the effective magnetic field acting on the current carries, through the increase of the susceptibility of the $4 f$ shell as $T$ is lowered. The role of the exchange field between $4 f$ spins and band carriers can be more directly accessible to analysis in the MR studies that will be discussed presently. 


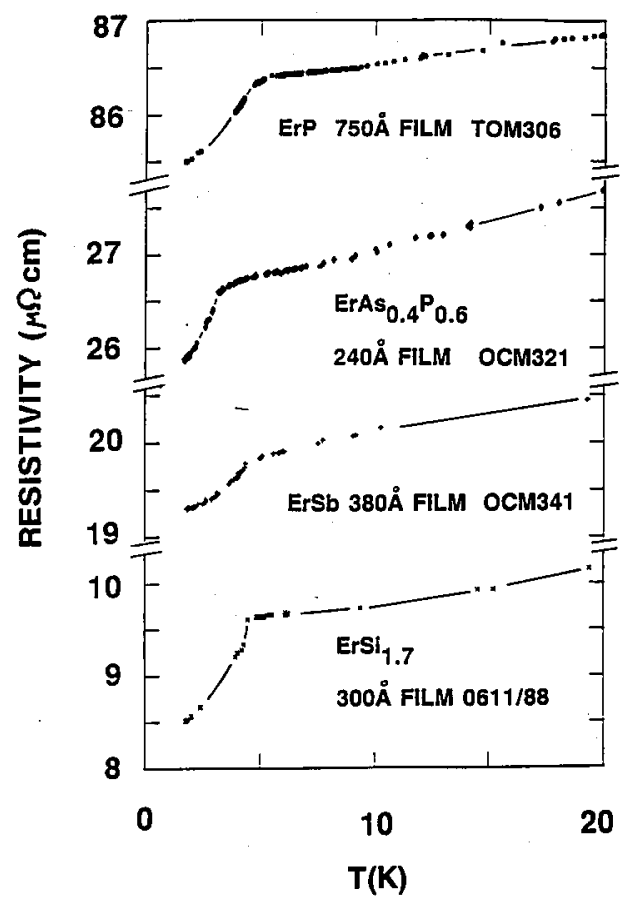

Fig. 4. Resistivity of several Er compounds near their ordering temperatures, $T_{c}$. The values are listed in Table II.

\section{Magnetoresistance}

Because of a strong coupling between the electrons and the localized magnetic moments of $\mathrm{Er}$ and because of relatively small electron mobility, the MR in $\mathrm{Er}$ silicide at low $T$ is dominated by the spin scattering effects. Because $\mathrm{Er}$ silicide is anisotropic the MR also shows anisotropies that are tractable to the anisotropies of the magnetic structure of the compound. Figure 5 shows MR measured in $\mathrm{ErSi}_{1.7}$ above and below the ordering temperature, $T_{\mathrm{c}}=4.5 \mathrm{~K}$. For the longitudinal case $(B \| I \perp c)$ at $T>T_{\mathrm{c}}$ the MR originates from the spin interactions alone. The analysis of the data in the paramagnetic region, where one observes a usual negative MR, is particularly simple. We fitted the data taken at $10 \mathrm{~K}$ and $20 \mathrm{~K}$, using a dependence following from the work of de Gennes and Friedel [16] on scattering of electrons by localized magnetic moments. They calculated the scattering cross section using the Born approximation and took the Heisenberg interaction Hamiltonian for spin-spin interactions. The scattering cross section thus obtained in proportional to $\left(S^{2}-\langle S\rangle^{2}\right)$. Further, we treated, for simplicity, the Er moments as spin $1 / 2$ particles, with the effective moment of $g \mu_{\mathrm{B}} / 2$. The resistivity can be then expressed as

$$
\rho=\rho_{0}+\rho_{S}\left[(3 / 4)-\tanh ^{2}\left(B g \mu_{\mathrm{B}} / 2 k T\right)\right]
$$


and two of the upper two curves in Fig. 5 show the result of the fitting procedure. The latter returned the values of the $g / 2$ of, respectively, $3.56 \mu_{\mathrm{B}}$ and $3.28 \mu_{\mathrm{B}}$, for

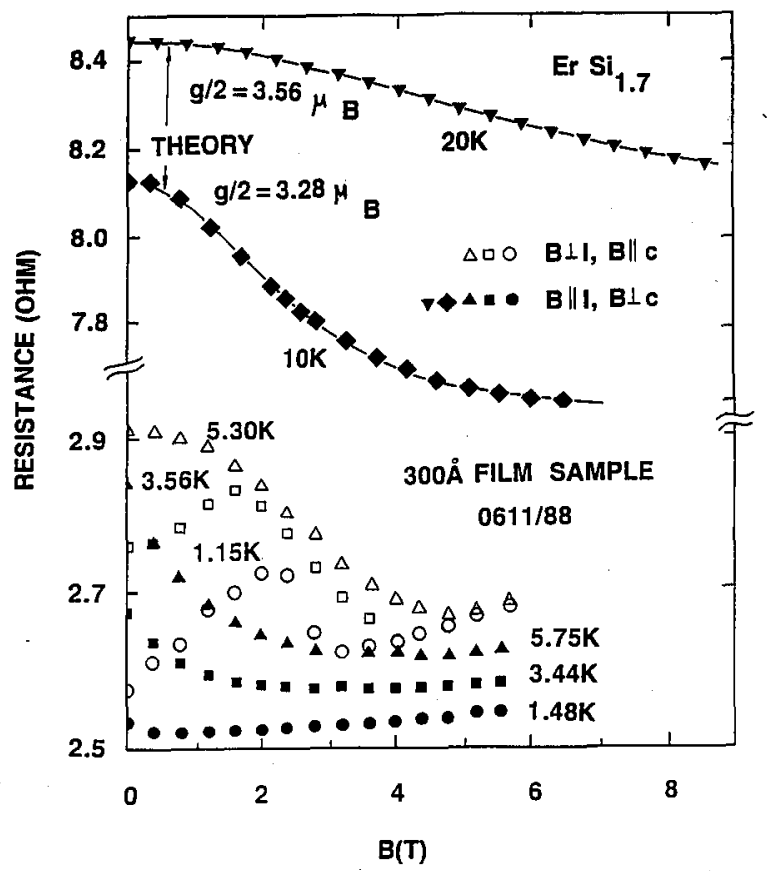

Fig. 5. Magnetoresistance for $B \| c \perp I$ ( $I$ is the current and $c$ the hexagonal axis) is a nonmonotonic function of $B$ below $T_{c}$. At $T>T_{c}$ the data are fitted using the de Gennes-Friedel theory [16].

the $20 \mathrm{~K}$ and the $10 \mathrm{~K}$ curves. An important feature of the fitting procedure was the use, for $B$ in Eq. (2), a sum of the applied $B_{\text {appl }}$ and the exchange $B_{\text {ex }}$ fields. The latter was assumed to be given by the Curie-Weiss law, with the ordering temperature $T_{\mathrm{c}}$. The expression used for the effective field $B$ in Eq. (2) was

$$
B=B_{\text {appl }}\left[1+T /\left(T-T_{\mathrm{c}}\right)\right] .
$$

At $T>T_{\mathrm{c}}$ the magnetic properties become isotropic.

The magnetic moment of the $\mathrm{Er}^{+3}$ can be directly measured as a high-field limit of magnetization for a proper orientation of the magnetic field. Magnetization $\mu$ normalised by the number of ions shown in Fig. 6 indicates that for $B \| c$, the saturation value of $\mu$ is about $3.7 \mu_{\mathrm{B}}$, the value corresponding within the error margin to the $g / 2$ values obtained from the diamagnetic MR fitting to Eq. (2). The fact that two independent measurements yielded the same value of $\mu$ for the $\mathrm{Er}^{+3}$ ion should be considered as one of the salient points of this study.

For $B \perp c$ and $\perp I, \mu$ raises to much higher values and the form of the $\mu(B)$ curve at lower values of $B$ suggests that metamagnetism transition (gradual 


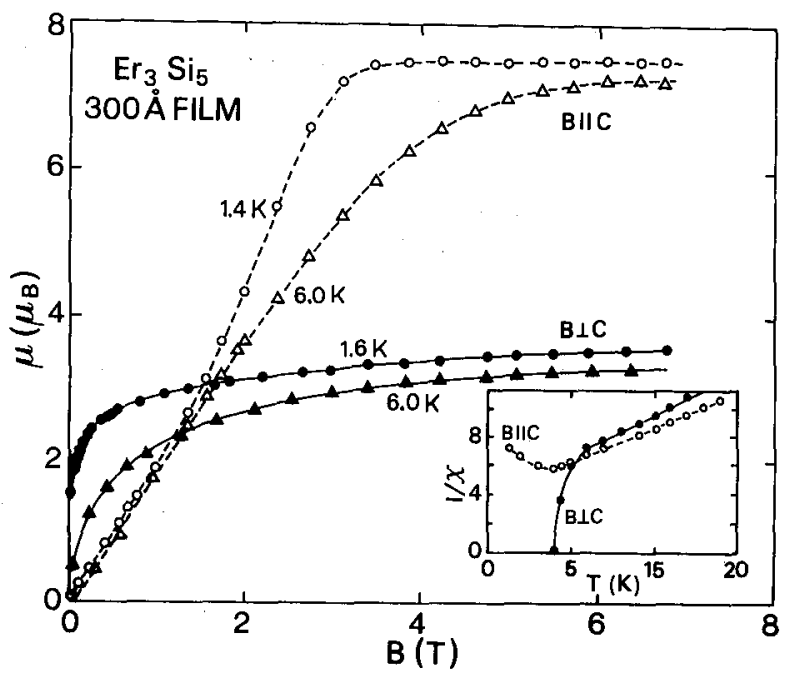

Fig. 6. Magnetization as a function of the magnetic field for two orientations of $B$ each for $T$ above and below $T_{c}$. Inset shows the inverse magnetic susceptibility for the same orientations of $B$.

change of antiparallel to parallel spin configuration) takes place. In order to account for the ensemble of the magnetization data a model of a magnetic structure of $\mathrm{Er}_{3} \mathrm{Si}_{5}$ was proposed. It was shown to account for the neutron scattering data obtained in a separate study on a powder $\mathrm{ErSi}_{1.7}$ sample [17]. The model is shown schematically in Fig. 7. Two, non-collinear magnetic components of the structure

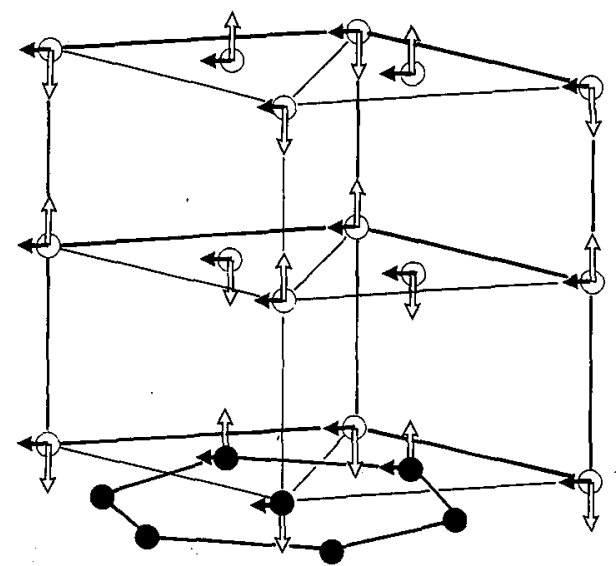

Fig. 7. Magnetic structure of Er silicide.

can be distinguished, one antiferromagnetic, along the $c$ axis $\left(5.7 \mu_{\mathrm{B}}\right.$ moments, 
white arrows) and second ferromagnetic (1.7 $\mu_{\mathrm{B}}$ moments, black arrows) lying in the (0001) plane. The magnetization data were then fitted using the Hamiltonian consisting of: (i) the crystal field (CF) terms, having a symmetry appropriate to the proposed hexagonal structure, and (ii) exchange terms. Four CF parameters and two (pairs) of exchange parameters were necessary for the fit, whose results are presented in Fig. 8. Subsequently, the $\mu(B)$ dependence was used for

a

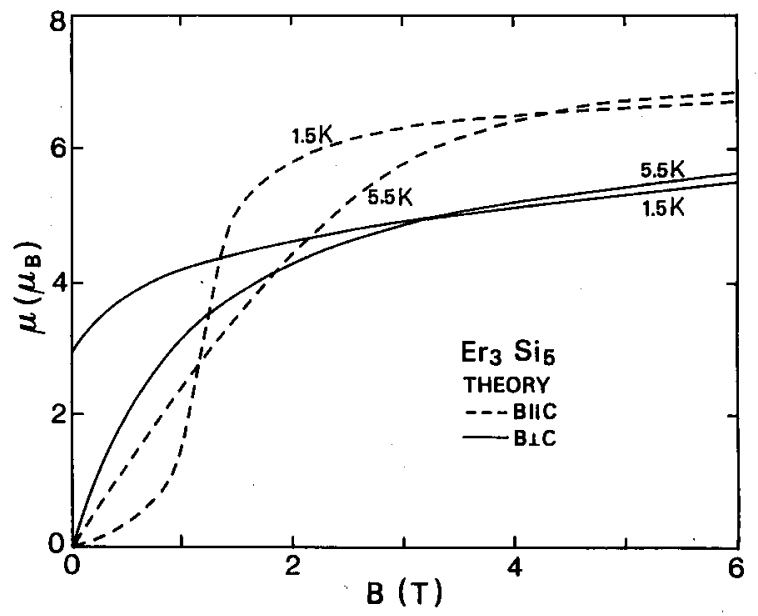

b

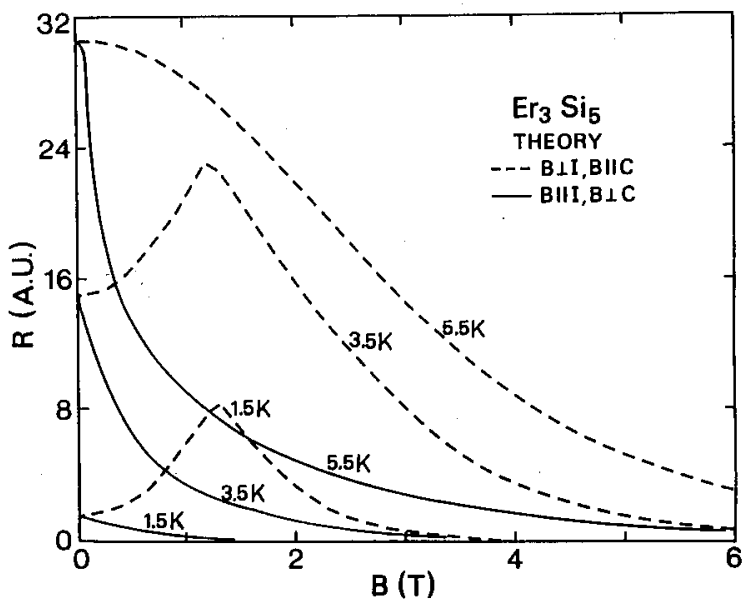

Fig. 8. (a) Magnetization computed in the molecular field model, for the field parallel and perpendicular to the $c$ orientation. (b) Magnetoresistance computed with the same parameters as those used in (a), according to the calculation of Yamada and Takada [18].

computing the transverse and longitudinal MR at various temperatures. For this purpose the results of theory developed by Yamada and Takada [18] (YT) for MR 
in the antiferromagnetic systems was used. YT calculate the MR from the spin correlation functions, using Heisenberg formalism to describe the interaction between the localized moments and electron spins. The discussion of the YT theory and its application for interpreting of our data is beyond the scope of this paper and the reader is referred to our former publication [19], where the outline of the work and the comparison between the experimental data and the calculated MR are presented, the final fit is presented in Fig 8. However, the knowledge of the magnetic structure (Fig. 7) allows for a qualitative discussion of the MR and the magnetization data in Er silicide presented in Figs. 5 and 6.

Let us note first that the exchange field can be evaluated from $T_{\mathrm{c}}$, because, approximately, $B_{\mathrm{ex}}=k T_{\mathrm{c}} / \mu$, giving a value of about $1.5 \mathrm{~T}$ for $\mu=3.7 \mu_{\mathrm{B}}$. Therefore if the magnetic field is swept through $1.5 \mathrm{~T}$ the antiparallel Er moments will start getting inverted and at sufficiently high fields the entire spin system will be aligned along $\boldsymbol{B}$. The system passes through a maximum of disorder at some point where resistance should be the largest. More precise description of the process is as follows. For uniaxial antiferromagnets one subset " $A$ " of moments originally "up", resides in the exchange field generated from the subset " $B$ " of originally "down" moments and vice versa. The external field applied, in say, "up" orientation reduces the effective field acting on the B subset (increasing that for "up" spins, that remain in that orientation). The energy separating the two possible states "up" and "down" of the subset diminishes linearly, as shown schematically in Fig. 9. When the doublet separation becomes comparable with $k T$, electrons

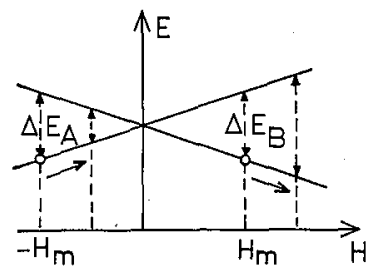

Fig. 9. Energy splitting for the two sublattices of the antiferro magnetic component of the magnetic structure. The applied field increases the energy splitting, given originally by the molecular field, for one sublattice and diminishes it for the other.

can be scattered, exchanging with the localized angular moments of Er, their energy, momentum, and spin. A comparison between the MR and the magnetization functions (Figs. 5 and 6 , or $8(\mathrm{a})$ and $8(\mathrm{~b})$ ) at the same temperatures shows that the maxima of the MR are located at the field values corresponding to the midrise point of the corresponding magnetization curves. This fact is consistent with the model of the effect: the maximum disorder means that about a half of the moments is flipped, hence magnetization is at its half increase. It should also be noted that the MR maxima move to higher values of $B$ with decreasing $T$, as required by the increase of $B_{\text {ex }}$ (cf. Eq. (2)).

The longitudinal MR at $T<T_{\mathrm{c}}$ was also satisfactorily accounted for by calculation; it is negative and featureless throughout the whole range of fields in 
Fig. 5. The absence of resistivity anomalies can be understood if one consults the model of the magnetic structure. The field applied in the basal plane of the hexagon inclines the antiferromagnetic moments in the applied field direction, after a rapid alignement of the ferromagnetic magnetic moments. At $T \ll T_{\mathrm{c}}$ the decrease of the resistance is small and the entire drop of resistance occurs in the field region where the magnetization increases rapidly, due to the alignment and saturation of the ferromagnetic component of the magnetic structure. The longitudinal MR is more pronounced at higher $T$, where the thermal spin fluctuations are stronger. The magnetic field suppresses the latter by increasing the splitting between the spin-up and the spin-down states. The longitudinal MR can be obtained from the sigmoidal MR curve of the longitudinal MR in the paramagnetic state (cf. Fig. 5, top), by shifting the origin by the amount $B_{\text {ex }}$. For this reason the longitudinal MR at $T<T_{\mathrm{c}}$ has a negative slope at the origin.

The study of MR in hexagonal materials is more interesting because the anisotropy of the structure allows for clear separation of the spin and other effects in transport phenomena. However a similar study was devoted to $\mathrm{Er}-\mathrm{V}$ compounds and Fig. 10 shows that the calculation accounts very well for the experimental data. Better agreement between the data and the calculation for the pnictides is

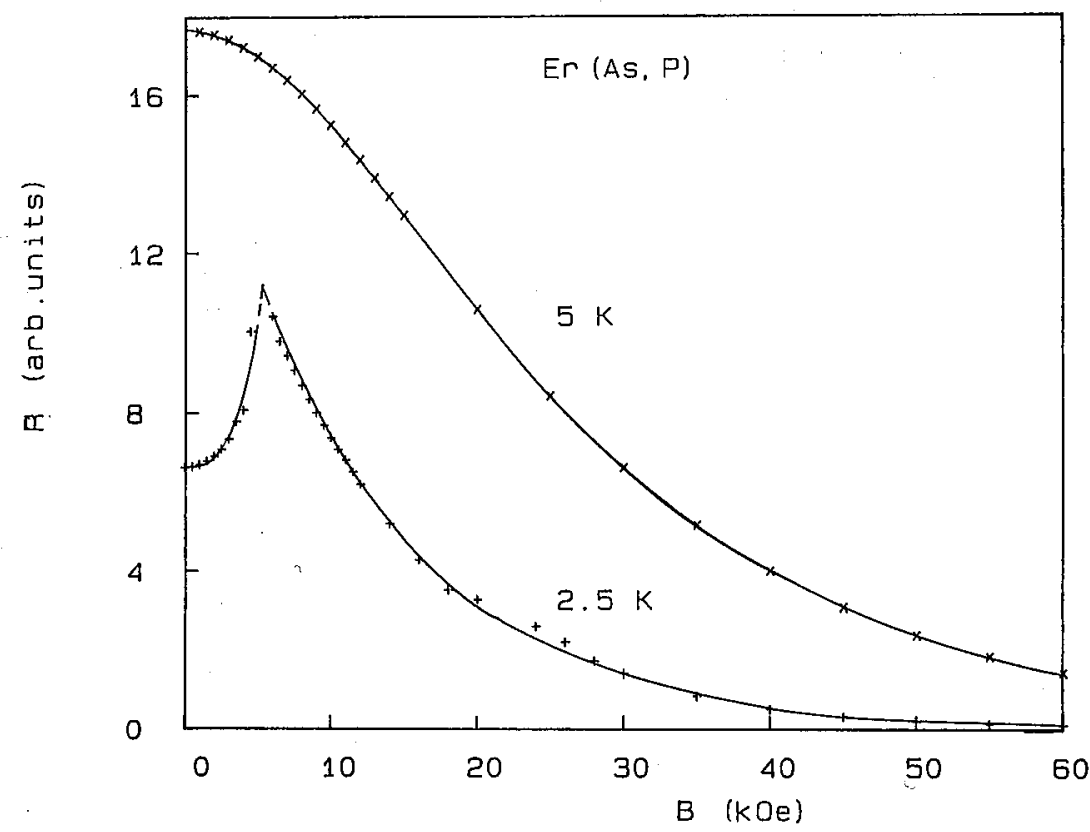

Fig. 10. Magnetoresistance computed for ErAso.4 $\mathrm{P}_{0.6}$.

due to the fact that several steps in the calculation are simpler and the fits of the magnetization require fewer adjustable parameters because of a simpler symmetry of the cubic system.

The applications of the RE compounds in microelectronics have not yet left 
the exploratory stage, mainly because of technological difficulties and the vacancy formation in the RE silicides can serve as an example. For this reason more research efforts are needed, considering the fact that the first RE compounds on semiconductors have been fabricated so very recently. On the other hand they offer a wealth of novel and exciting phenomena for studies of interactions between current carriers and localised magnetic ions.

\section{Acknowledgements}

This review presents mainly the results obtained in various laboratories in France: the Centre National d'Etudes des Télécommunications (CNET) Meylan $^{a}$ and CNET-Lannion ${ }^{b}$, laboratories of the Centre National de la Recherche Scientifique (CNRS) - Grenoble ${ }^{c}$ and High Field Magnet Laboratory (CNRS/MPI) ${ }^{\mathrm{d}}$. I list below the principal contributors to this work, with whom I had the privilege to collaborate, with my sincere thanks: F. Arnaud d'Avitaya ${ }^{\mathrm{a}}$, S. Auffret ${ }^{c}$, P.A. Badoz ${ }^{a}$, A. Briggs ${ }^{c}$, Y. Campidellia ${ }^{a}$ C. d'Anterroches ${ }^{a}$, J.Y. Duboz $^{a}, A$. Guivarc' $h^{b}$ (and his collegues from CNET-Lannion), B. Lambert ${ }^{c}$, W. Joss ${ }^{d}$, A. Perio ${ }^{a}$, and J. Pierre ${ }^{c}$. Discussions with S.J. Allen (Bellcore) have been most helpful in understanding the physics of the pnictides.

The travel grant obtained from the "Tempus" Office, Brussels, under contract No IMG-90-F-001 permitted the presentation of this review at the 20 th International School on the Physics of Semiconducting Compounds at Jaszowiec, Poland.

\section{References}

[1] V.A. Kasatkin, F.P. Kesamanly, V.G. Makarenko, V.F. Masterov, B.E. Samorukov, Fiz. Tekh. Poluprov. 14, 1832 (1980).

[2] H. Ennen, J. Schneider, G. Pomremke, A. Axman, Appl. Phys. Lett. 43, 943 (1983).

[3] Cf. e.g. Handbook on the Physics and Chemistry of Rare Earths, Eds. K.A. Gschneider, L. Eyring, North Holland, Amsterdam 1978.

[4] G.B. Printz,Science (in print).

[5] T. Sands, C.J. Palmstroem, J.P. Harbison, V.G. Keramidas, N. Tabatabaie, T.L. Cheeks, R. Ramesh, Y. Soilberberg, Mater. Sci. Rep. 5, 99 (1990).

[6] A. Le Corre, B. Gunais, A. Guivarc'h, D. Lecrosnier, J. Caulet, M. Minier, G. Ropars, P.A. Badoz, J.Y. Duboz, J. Crystal Growth 105, 234 (1990).

[7] C.J. Palmstroem, S. Mounier, T.G. Finstad, P.F. Miceli, Appl. Phys. Lett. 56, 382 (1990).

[8] J.E. Baglin, F.M. d'Huerle, C.S. Petersson, Appl. Phys. Lett. 36, 595 (1980).

[9] J.A. Knapp, S.T. Picraux, in MRS Symp. Proc., Vol. 54, North Holland, New York 1985, p. 261.

[10] F. Arnaud d'Avitaya, P.A. Badoz, Y. Campidelli, J.A. Chroboczek, J.Y. Duboz, A. Perio, J. Pierre, Thin Solid Films 184, 283 (1990). 
[11] F.H. Kaatz, W.H. Graham, J. Van der Spiegel, W. Joss, J.A. Chroboczek, Proc. 37th Symp. Am. Vac. Soc., Toronto 1990 (in print).

[12] R. Baptist, S. Ferrer, G. Grenet, H.C. Poon, Phys. Rev. Lett. 64, 311 (1990).

[13] S.J. Allen, N. Tabatabaie, C.J. Palmstroem, G.W. Hull, T. Sands, F. DeRosa, H.L. Gilchrist, K.C. Garrison, Phys. Rev. Lett. 62, 2309 (1989).

[14] A. Guivarc'h, A. Le Corre, J. Gaulet, B. Guenais, M. Minier, G. Ropars, P.A. Badoz, J.Y. Duboz, in MRS Symp. Proc., Vol. 160, North Holland, New York 1990, p. 331.

[15] S.J. Allen, F. de Rosa, C.J. Palmstroem, A. Zrener, (submitted to Phys. Rev. B).

[16] P.G. de Gennes, J. Friedel, J. Phys. Chem. Solids 4, 71 (1958).

[17] S. Auffret, J. Pierre, B. Lambert, J.L. Soubeyroux, J.A. Chroboczek, Physica B 162, 271 (1990).

[18] H. Yamada, S. Takada, J. Phys. Soc. Jpn. 34, 51 (1973).

[19] J.A. Chroboczek, A. Briggs, W. Joss, S. Auffret, J. Pierre, Phys. Rev. Lett. 66, 790 (1991). 